\title{
Forschungsinfrastruktur für empirische Sozialforschung in Österreich - Zwischen Anspruch und Bedarf
}

\author{
Dimitri Prandner • Otto Bodi-Fernandez • Franz Höllinger • \\ Ahmed Tabakovic
}

(C) Der/die Autor(en) 2019

Zusammenfassung Sozialwissenschaftliche Forschung wird in zunehmendem Maße durch Forschungsinfrastrukturen unterstützt, die empirische Forschungsdaten sammeln und zur Nachnutzung für die wissenschaftliche Gemeinschaft bereitstellen. 2016 wurde zu diesem Zweck AUSSDA - The Austrian Social Science Data Archive gegründet. Im ersten Abschnitt dieses Forschungsberichts werden einige wichtige Gründe erörtert, die für den Aufbau und die Nutzung derartiger Einrichtungen sprechen. Im zweiten Teil werden die Ergebnisse einer Mapping-Studie präsentiert, in der versucht wurde, den potenziellen Nutzerkreis dieses Datenarchivs an österreichischen Universitäten zu erfassen. Hierbei wurden Profile von etwa 3000 österreichischen SozialwissenschaftlerInnen erstellt sowie mehr als 800 empirische Forschungsprojekte erfasst und nach relevanten Merkmalen kategorisiert.

Schlüsselwörter Datenarchiv · Sozialwissenschaften · Empirische Sozialforschung $\cdot$ Wissenschaftsforschung

\footnotetext{
D. Prandner $(\bowtie)$

Institut für Soziologie - Abteilung für Empirische Sozialforschung, Johannes-Kepler-Universität Linz, Altenbergerstraße 69, 4040 Linz, Österreich

E-Mail: Dimitri.Prandner@jku.at

O. Bodi-Fernandez

Centrum für Sozialforschung, Karl-Franzens-Universität Graz, Universitätsstraße 15, 8010 Graz, Österreich

E-Mail: Otto.Bodi@uni-graz.at

\section{F. Höllinger}

Institut für Soziologie, Karl-Franzens-Universität Graz, Universitätsstraße 15, 8010 Graz, Österreich E-Mail: Franz.Hoellinger@uni-graz.at
}

\section{A. Tabakovic}

Fachbereich Kommunikationswissenschaft, Universität Salzburg, Rudolfskai 42, 5020 Salzburg, Österreich

E-Mail: Ahmed.Tabakovic@stud.sbg.ac.at 


\title{
Infrastructure for empirical social research in Austria—between expectations and demand
}

\begin{abstract}
There is a trend to support social science research via national research infrastructures, which ensure that research data is stored safely and available for further use. In 2016, AUSSDA-The Austrian Social Science Data Archive-was established to fulfill this role in Austria. The first part of the research note illustrates the benefits such an institution offers, while the second part gives insight into potential users of such an infrastructure. Using data from a recent research project it illustrates that since 2016 around 3000 Austrian social scientists conducted more than 800 empirical research projects.
\end{abstract}

Keywords Data archiving $\cdot$ Social Sciences $\cdot$ Empirical social research $\cdot$ Science studies

\section{Empirische Sozialforschung als Kooperationsprojekt - Gründe für die Einrichtung und Nutzung von Datenarchiven}

Vor dem Hintergrund der sogenannten ,datafication of society“ (vgl. u. a. Lycett 2013 oder Baack 2015) kam es seit den 1980er-Jahren auch in den Sozialwissenschaften zu einem rapiden Anstieg von Datenbeständen, die an universitären und außeruniversitären Forschungseinrichtungen generiert und bearbeitet werden (Couldry 2014). Ein kleiner Teil dieser Forschung, insbesondere die Erhebungen von nationalen und internationalen Forschungskooperativen, wie beispielsweise die Allgemeine Bevölkerungsumfrage für Sozialwissenschaften in Deutschland (ALLBUS), der European Value Survey (EVS) und das International Social Survey Programme (ISSP), wurde bereits bisher der gesamten sozialwissenschaftlichen Forschergemeinschaft zur Verfügung gestellt. Der Großteil der empirischen Forschungsdaten war jedoch bislang nur den beteiligten ForscherInnen sowie deren informellen Netzwerken zugänglich; vielfach betrachten ForscherInnen ,ihre“ Daten als Eigentum, zu dem niemand anderer Zugang haben sollte (Bauer et al. 2015a).

Es gibt jedoch eine Reihe von Gründen, die dafürsprechen, Forschungsdaten zu archivieren und zur Nachnutzung bereitzustellen (siehe u. a. Heidorn 2008; Borgman 2012; Fecher et al. 2015; Prandner und Raffetseder 2018): Publizierte Datensätze sind zitierfähig und erhöhen die Sichtbarkeit sowohl der Daten als auch der zugehörigen Forschungsleistungen und Publikationen. Das Teilen und Bereitstellen von Forschungsdaten und der entsprechenden Dokumentation bedeutet zudem auch, den Anforderungen von Förderrichtlinien und guter wissenschaftlicher Praxis hinsichtlich Transparenz und Nachhaltigkeit gerecht zu werden. Zeitgleich haben die Erwartungen hinsichtlich Open Science in den letzten Jahren deutlich zugenommen: Fördergeber und Journals verlangen zunehmend Open Access nicht nur bei Publikationen, sondern explizit auch bei Forschungsdaten. So ist etwa der aktuellen OpenAccess-Policy des FWF (2018) zu entnehmen: ,Für Forschungsdaten, die den wissenschaftlichen Publikationen des Projekts zugrunde liegen, ist der offene Zugang verpflichtend. Das sind alle Daten, die zur Reproduktion und Überprüfbarkeit der 
Ergebnisse der Publikationen erforderlich sind, einschließlich der zugehörigen Metadaten. Diese Daten sollen schnellstmöglich veröffentlicht werden, jedoch spätestens zusammen mit der entsprechenden wissenschaftlichen Publikation.“

Mit der Gründung von Austrian Social Science Data Archive (AUSSDA) wurde 2016 an der Universität Wien eine zentrale Anlaufstelle für die Archivierung sozialwissenschaftlicher Daten in Österreich eingerichtet. AUSSDA ist zugleich auch Mitglied des Consortium of European Social Science Data Archives (CESSDA), das seit seiner Gründung 1976 als Kooperationsplattform für Datenarchive in Europa agiert (Prandner und Raffetseder 2018, S. 238); durch die Anbindung von AUSSDA an CESSDA wird es in absehbarer Zukunft möglich sein, die in Österreich archivierten Daten in allen CESSDA-Archiven und somit auf internationaler Ebene zu finden und zu nutzen.

Mit der Archivierung der Daten bei AUSSDA werden die oben skizzierten Anforderungen an Transparenz und Nachhaltigkeit erfüllt, da Datensätze durch persistente Identifikationsnummern (DOI) zitierbar gemacht werden, und die Wiederverwendung der Daten durch entsprechende Lizenzmodelle (z. B. Open Access- oder Scientific Use-Lizenzen) sichergestellt wird. Der Aufgabenbereich von AUSSDA beschränkt sich aber nicht nur auf die Archivierung und Bereitstellung von Forschungsdaten. Als zentrale Infrastruktureinrichtung für die Sozialwissenschaften in Österreich wirkt das Archiv als Plattform, die sozialwissenschaftliche Forschungsergebnisse innerhalb der Forschungscommunity und darüber hinaus sichtbarer macht und somit Möglichkeiten schafft, auf vorhandener Forschung aufzubauen und neue Kooperationen einzugehen. Diese Rolle soll u. a. auch durch Workshops zu Datenmanagement und Forschungsmethodik genauso wie durch das Bereitstellen von Open Educational Ressources (frei zugängliche Lehrmaterialien) wahrgenommen werden.

\section{Potenziale in der aktuellen sozialwissenschaftlichen Forschungslandschaft}

Im Kontext der Einrichtung eines sozialwissenschaftlichen Datenarchivs stellt sich auch die Frage, in welchem Ausmaß ein Nutzungspotenzial in der nationalen Community gegeben ist. Um das Nutzungspotenzial $\mathrm{zu}$ erfassen, ist es unabdingbar, besser über die Struktur der Sozialwissenschaften in Österreich Bescheid zu wissen. Jedoch gibt es bislang keine zuverlässigen Quellen über Umfang und Struktur des Felds der sozialwissenschaftlichen Disziplinen in Österreich. Die existierenden Dokumentationen, die über verschiedene Kanäle verfügbar sind - Wissensbilanzen oder Statistiken der Hochschulen, Forschungseinrichtungen und Ministerien sind sehr heterogen und in vielerlei Hinsicht wenig transparent. Diese Situation ergibt sich zum Teil dadurch, dass die Verfasser bzw. Auftraggeber dieser Dokumentationen jeweils unterschiedliche Zielsetzungen verfolgen und dementsprechend unterschiedliche Leistungsindikatoren erheben. Zudem ist die Zuordnung einzelner Disziplinen zum Bereich der Sozialwissenschaften sehr uneinheitlich; es werden hier aktuell unterschiedliche Ansätze verfolgt: 
Auf der Ebene der Universitäten werden sozialwissenschaftliche Disziplinen in unterschiedlicher Weise gebündelt und zum Teil mit geistes- und kulturwissenschaftlichen, zum Teil mit wirtschaftswissenschaftlichen Disziplinen zu Fakultäten zusammengefasst (vgl. Bauer et al. 2015a). Zudem ordnen sich einzelne Studienrichtungen selbst je nach Standort unterschiedlichen Wissenschaftsbereichen zu. So wurde beispielsweise das Soziologiestudium an bestimmten Standorten als geisteswissenschaftliche, an anderen Standorten als sozial- und wirtschaftswissenschaftliche Studienrichtung eingerichtet. Auch in Publikationen des Bundesministeriums für Bildung, Wissenschaft und Forschung werden Studienzweige in unterschiedlicher Weise in Hauptgruppen zusammengefasst (vgl. z. B. BMFWF 2017). In der Wissenschaftsstatistik der Statistik Austria wie auch beim österreichischen Fonds zur Förderung der Wissenschaftlichen Forschung (FWF) werden die Wissenschaftsdisziplinen nach der Österreichischen Systematik der Wissenschaftszweige klassifiziert. Zur Hauptkategorie 5 - Sozialwissenschaften - zählen hier folgende Fachbereiche: Psychologie (501), Wirtschaft (502), Pädagogik (503), Soziologie (504), Rechtswissenschaften (505), Politikwissenschaften (506), Humangeographie (507), Medienund Kommunikationswissenschaften (508) sowie sonstige sozialwissenschaftliche Fächer (509) wie beispielsweise Disability Studies, Evaluationsforschung, Minderheitenforschung oder Wissenschaftsforschung. Die Größe des Feldes der Sozialwissenschaften variiert somit sehr stark, je nachdem, welche Wissenschaftszweige dieser Kategorie zugeordnet werden.

Bei der Abgrenzung des Erhebungsbereichs für unsere Studie Mapping the Field diente die Kategorie Sozialwissenschaften der ÖFOS 2012 Wissenschaftsklassifikation als Ausgangspunkt. Drei der dort angeführten Wissenschaftszweige, Psychologie, Rechtswissenschaften und Humangeographie, wurden jedoch nicht in die Erhebung einbezogen. Der Ausschluss dieser Fachbereiche erschien deshalb sinnvoll, weil die entsprechenden Institute an Österreichs Universitäten in der Regel nicht in sozialwissenschaftliche Fakultäten integriert sind und weil sich viele ihrer VertreterInnen selbst in erster Linie als Natur- bzw. als RechtswissenschaftlerInner und nicht als SozialwissenschaftlerInner verstehen; im Fall der Rechtswissenschaften auch deshalb, weil hier nur relativ wenig empirische Sozialforschung betrieben wird, die für die Datenarchivierung bei AUSSDA relevant ist.

Hinsichtlich der untersuchten Organisationen beschränkten wir uns in der hier präsentierten ersten Phase des Projekts auf öffentliche und private Universitäten sowie jene Fachhochschulen, die aktuell durch die nationale Qualitätssicherungsagentur AQ Austria ${ }^{1}$ akkreditiert werden. Weitere Einrichtungen, an denen sozialwissenschaftliche Lehre und Forschung stattfindet, wurden aufgrund der zeitlich und finanziell begrenzten Ressourcen des Projekts vorerst ausgespart. Dies bedeutetet, dass Pädagogische Hochschulen und andere Einrichtungen, wie beispielsweise private und öffentliche Forschungsinstitute (z. B. OeAW, WIFO, IHS), nicht erfasst wurden und sich die folgenden Ausführungen rein auf Universitäten und Fachhochschulen beziehen, wo auch die zentralen NutzerInnen des Datenarchivs verortet werden. Die Datenerfassung wurde im September und Oktober 2018 mittels einer eigenständigen Onlinerecherche zu Projekten und Personen durchgeführt.

\footnotetext{
1 Siehe https://www.aq.ac.at/de/.
} 
Die den Sozialwissenschaften zuzurechnenden Personen wurden in mehreren Stufen erhoben. In einem ersten Schritt wurden das Personal von den Instituten und Fachbereichen sozialwissenschaftlicher Fakultäten und Organisationseinheiten über die jeweiligen Homepages erfasst und entsprechend den ÖFOS 2012-Kategorien der Gruppe 5 zugeordnet.

Im nächsten Schritt wurden Institute und Fachbereiche, die nicht unter die ausgewählten ÖFOS 2012-Kategorien fallen, aber an den Fakultäten und Organisationseinheiten der Sozialwissenschaften angesiedelt sind, erfasst. Schlussendlich wurden die Selbstbeschreibungen von fachnahen Disziplinen und Instituten ermittelt und jene Organisationseinheiten, die sich in ihrer Selbstwahrnehmung als Sozialwissenschaft verstehen, in die Erhebung aufgenommen. So konnten beispielsweise VertreterInnen aus der Psychologie (ÖFOS 2012, Kategorie 501) und der Rechtswissenschaften (ÖFOS 2012, Kategorie 505), die sich explizit den Sozialwissenschaften zuordnen, auch erfasst werden. Personen und Projekte, die nicht eindeutig den Sozialwissenschaften zuzuordnen sind, wurden ausgespart, um eine potenzielle Übererfassung zu verhindern.

Mit dieser Vorgehensweise wurden 2940 Personen an Fachhochschulen sowie öffentlichen und privaten Universitäten identifiziert. Für jede erfasste Person wurde codiert, ob sie seit 2016 an einem empirischen Projekt mitgearbeitet hat, ob sie in dem Zeitraum in der angewandten Methodenlehre - Kurse zu Methoden der empirischen Sozialforschung und Datenanalyse, Forschungspraktika, Forschungsseminare etc. - tätig war und ob die Lehrtätigkeit einen quantitativen oder/und qualitativen Schwerpunkt hatte. Als zusätzliche Kontextvariablen wurden Berufs- und Bildungstitel, Karrierestufe sowie Geschlecht erfasst. Auffällig waren hier vor allem die uneinheitliche Verwendung von Berufs- und Bildungstiteln, da sich Angaben je nach Institution stark unterscheiden, was dazu führte, dass bisher auf eine diesbezügliche Detailauswertung verzichtet wurde.

In einem zweiten Schritt wurden die empirischen Forschungsprojekte der jeweiligen Fachbereiche und Institute systematisch erfasst, die seit 2016 durchgeführt wurden. Dazu wurden die öffentlich zugänglichen Forschungsdokumentationsdatenbanken, die Jahresberichte und Homepages der Organisationseinheiten herangezogen; die hier vorgefundenen Daten wurden mit den Projektdokumentationen des FWF, der FFG und des Jubiläumsfonds der OeNB abgeglichen. Die erstellte Datenbank umfasst alle Projekte, aus deren Beschreibung nachvollziehbar hervorgeht, dass im Rahmen des Projekts empirische Daten erhoben und/oder analysiert wurden. Eigenprojekte und Projekte ohne Förderung wurden ausgespart, da diese nicht einheitlich registriert wurden. Zusätzlich musste eine Bereinigung stattfinden, da manche Systeme auch beantragte Projekte ausgeben, die nicht genehmigt wurden. So konnten schlussendlich 808 Projekte in den Datensatz aufgenommen und Personen zugeordnet werden. Tab. 1 bietet einen Überblick über die erhobenen Variablen, die für diesen Forschungsbericht von Relevanz sind.

Das skizzierte Vorgehen führt sowohl auf Personen- wie auch auf Projektebene zu konservativen Schätzungen mit einem Potenzial zur Untererfassung; nicht alle Projekte sind in öffentlich zugänglichen Datenbanken verzeichnet und beim Personalstand war man auf die Vollständigkeit der Angaben auf den Homepages angewiesen. Vor allem bei den privaten Universitäten waren oftmals Informationen 
Tab. 1 Grundlegende Variable zu den österreichischen SozialwissenschaftlerInnen $(N=2940)$

\begin{tabular}{|c|c|c|}
\hline Variable & Ausprägungen & Anteil (\%) \\
\hline \multirow{3}{*}{$\begin{array}{l}\text { Einrichtungsart } \\
(N=2940)\end{array}$} & Öffentliche Universitäten & 70,3 \\
\hline & Private Universitäten & 2,4 \\
\hline & Fachhochschulen & 27,3 \\
\hline \multirow[t]{3}{*}{$\begin{array}{l}\text { Karrierestufe } \\
(N=2795)^{\mathrm{a}}\end{array}$} & $\begin{array}{l}\text { Leitungs- und Führungspositionen (Univ.-Prof. bzw. } \\
\text { o.Univ.Prof./LeiterInnen der Organisationseinheit) }\end{array}$ & 12,6 \\
\hline & $\begin{array}{l}\text { Etabliertes wissenschaftliches Personal (Ass./Asc.-Prof., } \\
\text { FH-Prof., Post-Doc, Senior Lec./Scien., Vertragsbediens- } \\
\text { tete) }\end{array}$ & 43,8 \\
\hline & $\begin{array}{l}\text { Wissenschaftlicher Nachwuchs (Wissenschaftliche Mitar- } \\
\text { beiterInnen, ProjektmitarbeiterInnen, Pre-Doc.) }\end{array}$ & 43,6 \\
\hline \multirow{2}{*}{$\begin{array}{l}\text { Geschlecht } \\
(N=2940)\end{array}$} & Männlich & 55,2 \\
\hline & Weiblich & 44,8 \\
\hline $\begin{array}{l}\text { Projektbeteiligung } \\
(N=2940)\end{array}$ & $\begin{array}{l}\text { Zwischen } 2016 \text { und } 2018 \text { an empirischen Forschungspro- } \\
\text { jekten beteiligt gewesen }\end{array}$ & 22,0 \\
\hline \multirow{3}{*}{$\begin{array}{l}\text { Methodenunterricht } \\
(N=2881 ; \\
\text { Mehrfachcodierung } \\
\text { möglich) }\end{array}$} & $\begin{array}{l}\text { Zwischen } 2016 \text { und } 2018 \text { Lehrveranstaltungen in der } \\
\text { angewandten Methodenlehre abgehalten }\end{array}$ & 19,7 \\
\hline & $\begin{array}{l}\text { Zwischen } 2016 \text { und } 2018 \text { Lehrveranstaltungen in der } \\
\text { angewandten Methodenlehre abgehalten, die einen expli- } \\
\text { ziten Fokus auf qualitative Sozialforschung hatte }\end{array}$ & 7,3 \\
\hline & $\begin{array}{l}\text { Zwischen } 2016 \text { und } 2018 \text { Lehrveranstaltungen in der } \\
\text { angewandten Methodenlehre abgehalten, die einen expli- } \\
\text { ziten Fokus auf quantitative Sozialforschung hatte }\end{array}$ & 8,3 \\
\hline
\end{tabular}

\footnotetext{
aAbweichung, da nicht zu allen Personen die notwendigen Informationen verfügbar waren

${ }^{\mathrm{b}}$ Es wurden Eigenprojekte bzw. In-Kind-Projekte - also Projekte, die ohne externe Beteiligung oder Förderung durchgeführt werden, aufgrund ihrer uneinheitlichen Klassifizierung in den Forschungsdatenbanken ausgespart
}

zu Forschungsprojekten oder Lehrveranstaltungen nicht offen zugänglich. Dennoch konnten anhand der vorhandenen und zugänglichen Dokumentation für 2149 Personen vollständige Profile erstellt werden.

\subsection{Bisherige Ergebnisse der Studie im Überblick}

Betrachtet man die konkreten Ergebnisse, zeigt sich auf einer deskriptiven Ebene bereits eine Vielzahl von relevanten Informationen. Der erste Schritt für diese Analysen war eine Klassifikation der erfassten Personen $(n=2149)$ in vier Gruppen (siehe auch Tab. 2):

1. Die erste Gruppe umfasst jene, die sowohl im Rahmen von empirischen Projekten forschen, als auch in der angewandten Methodenlehre tätig waren. Insgesamt fallen $9 \%$ bzw. 195 Personen in diese Kategorie.

2. Die zweite Gruppe enthält jene Forschenden, die in empirischen Projekten tätig sind, aber nicht lehren, und macht $13 \%$ bzw. 281 der erfassten Personen aus, zu denen vollständige Profile vorliegen. 
Tab. 2 Klassifizierung der WissenschaftlerInnen bezüglich der Arbeit mit empirischen Daten $(n=2149)$

\begin{tabular}{llll}
\hline & & \multicolumn{2}{l}{ Anbieter von Kursen in angewandter Methodenlehre } \\
& & Ja & Nein \\
\hline $\begin{array}{l}\text { Durchführung von } \\
\text { empirischen }\end{array}$ & Ja & I - Forschende und Lehrende & II - Forschende \\
Forschungsprojekten & \multirow{2}{*}{ Nein } & $(9,1 \%)$ & $(13,1 \%)$ \\
& & III - Lehrende & IV - Potenzielle NutzerInnen \\
& $(17,4 \%)$ & $(60,5 \%)$ \\
\hline
\end{tabular}

3. Zur dritten Gruppe zählen 373 Personen, die im Bereich der empirischen Sozialforschung lehren, aber nicht an entsprechenden Projekten beteiligt sind. Ihr Anteil beträgt $17 \%$.

4. Die vierte Gruppe ist schlussendlich jener Personenkreis, der weder in der Lehre noch in empirischen Forschungsprojekten involviert ist. Sie sind mit $60 \%$ die größte Gruppe. Sie werden als potenzielle NutzerInnen für AUSSDA bezeichnet, da sie zwar nicht direkt als DatenlieferantInnen oder NutzerInnen für Daten im Bereich der Lehre gesehen werden können, aber im Rahmen ihrer wissenschaftlichen Tätigkeit ein Potenzial hinsichtlich Sekundarauswertungen von Daten darstellen.

Die Verteilung der Wissenschaftler auf diese vier Kategorien unterscheidet sich stark nach den Wissenschaftsbereichen der ÖFOS-Klassifikation: Während in den Wirtschaftswissenschaften und den Politikwissenschaften mehr als die Hälfte in die Gruppe IV fallen, sind es bei den Fächern, die den Erziehungswissenschaften, der Soziologie, der Medien- und Kommunikationswissenschaft sowie den anderen Sozialwissenschaften - in dieser Darstellung aufgrund der geringen Fallzahl der Einzeldisziplinen geschuldet - deutlich weniger. Das dort tätige Personal ist stärker an empirischen Forschungsprojekten und angewandter Methodenlehre beteiligt (siehe Abb. $\left.1^{2}\right)$.

Auch die Hierarchiestufen unterscheiden sich deutlich in Hinblick auf unsere Klassifizierung. Je etablierter die Personen im wissenschaftlichen System sind, desto eher fallen sie in die Gruppen I bis III. Der wissenschaftliche Nachwuchs ist deutlich seltener in der angewandten Lehre tätig und in Forschungsprojekte involviert (siehe Abb. 2). Trennt man den Nachwuchs weiter zwischen Personen auf Planstellen und ProjektmitarbeiterInnen auf, wird der Unterschied nochmals deutlicher. ProjektmitarbeiterInnen, die an empirischen Projekten arbeiten, müssen logischerweise Teil der Gruppen I oder II sein.

Ebenso verteilen sich die Gruppen je nach Organisationstypus unterschiedlich. An den Fachhochschulen unterrichten deutlich weniger Personen im Bereich der angewandten Methodenausbildung als an den öffentlichen Universitäten. Dies ist sowohl den höheren Lehrverpflichtungen als auch den Studienplänen zuzuschreiben. So unterrichten Personen, die im Methodensektor an Fachhochschulen tätig sind,

\footnotetext{
2 Personen, die in den Selbstzuschreibungen und Organisationszuschreibungen als SozialwissenschaftlerInnen zu identifizieren waren, aber in den Kategorien ÖFOS 2012 501, 505 oder 507 tätig sind, wurden aufgrund der geringen Fallzahlen mit Personen aus Fächern ,,andere Sozialwissenschaften“ in der Überkategorie 509 zusammengefasst.
} 


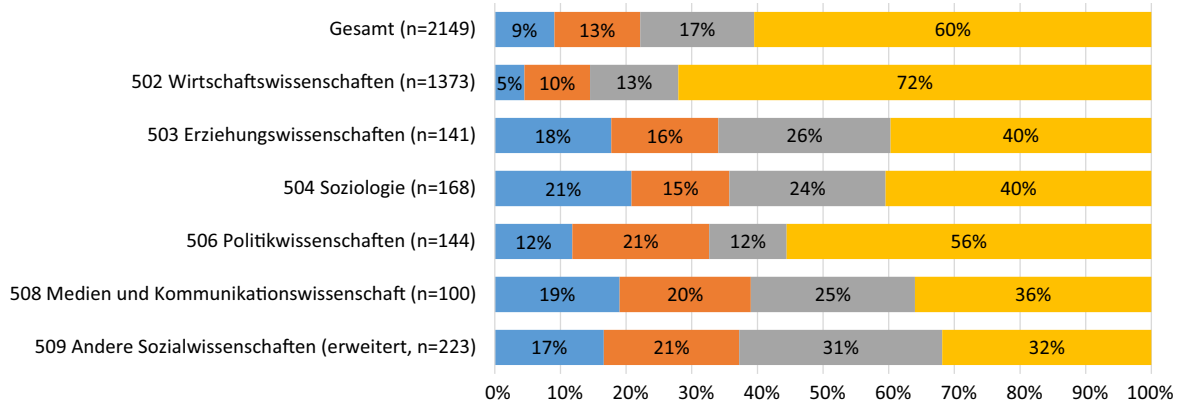

I- Forschende und Lehrende $\quad$ II-Forschende $\quad$ III-Lehrende $\quad$ IV - Potentielle NutzerInnen

Abb. 1 Arbeit mit empirischen Forschungsdaten nach Disziplinen

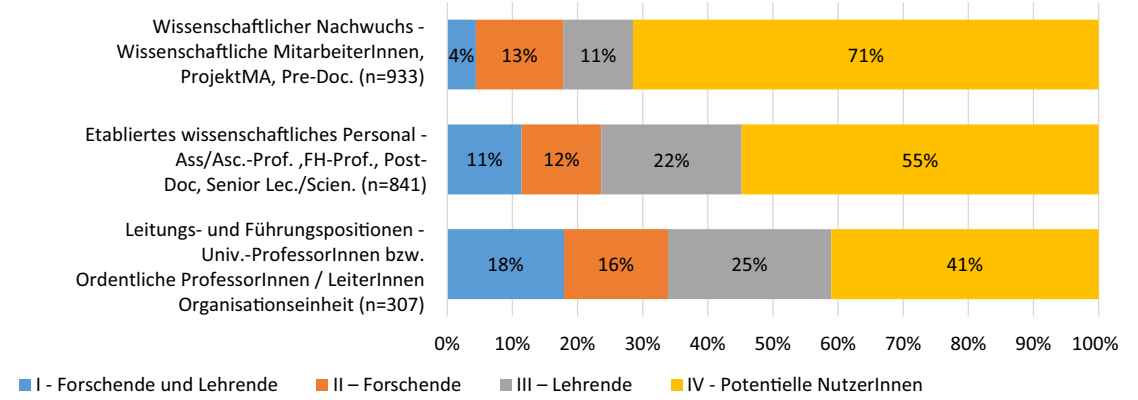

Abb. 2 Empirische Forschung und Lehre nach Karrierefortschritt

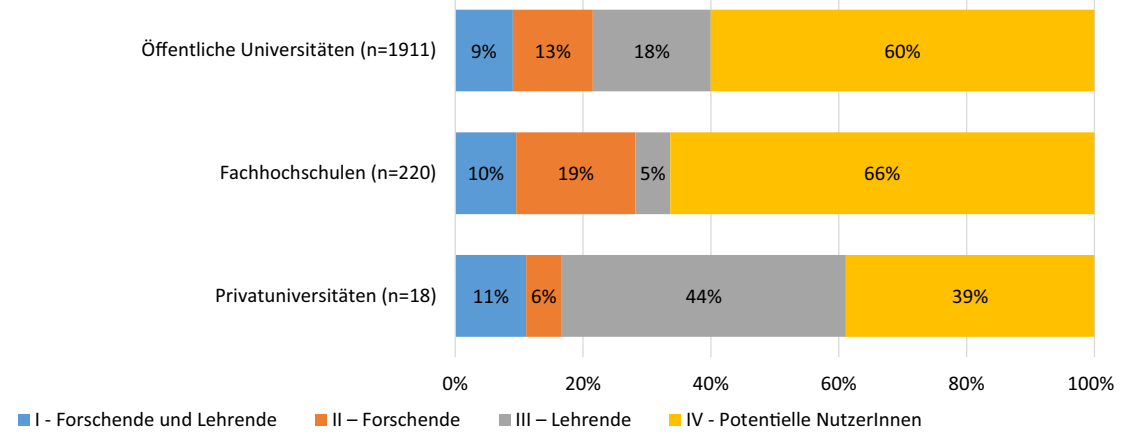

Abb. 3 Empirische Forschung und Lehre nach Organisationstyp

oftmals mehrere Kurse und können so große Teile der Methodenausbildung mit einer geringeren Kopfzahl abdecken. Zeitgleich müssen aber auch mehr - zusätzliche - MitarbeiterInnen rein für empirische Projekte angestellt und finanziert werden. Aufgrund der geringen Anzahl von Personen, die an privaten Universitäten tätig sind und zu denen vollständige Profile erstellt werden konnten, sind hier keine zuverlässigen Aussagen möglich (siehe Abb. 3). 


\section{Erkenntnisse aus dem Datenmaterial und die Notwendigkeit für Infrastruktur}

Unsere Erhebung der Größe des Felds der empirischen Sozialforschung und der Zahl der WissenschaftlerInnen, die an sozialwissenschaftlichen Instituten der österreichischen Universitäten und Fachhochschulen tätig sind, brachte folgende Hauptergebnisse: Seit der Gründung von AUSSDA im Jahr 2016 wurden an den untersuchten Instituten mehr als 800 empirische Forschungsprojekte durchgeführt, die potenziell im österreichischen Datenarchiv AUSSDA archiviert werden könnten. Die Frage, ob es sich lohnt, die im Projekt erhobenen Daten in einem nationalen Datenarchiv zu archivieren, und ob die Daten für eine Archivierung geeignet sind, muss in jedem Einzelfall eigens abgeklärt werden. Obwohl der österreichische Forschungsförderungs-Fonds FWF die Archivierung der erhobenen empirischen Forschungsdaten schon seit Jahren empfiehlt und fordert, wird jedoch bisher nur ein verschwindend kleiner Teil der in Österreich erhobenen sozialwissenschaftlichen Forschungsdaten in adäquater Weise in einem öffentlichen Datenarchiv abgelegt und für eine Sekundärnutzung zur Verfügung gestellt. Die 2015 von Bauer et al. (2015b, S. 570) getroffene Feststellung, dass in den Sozialwissenschaften Forschungsdaten vergleichsweise selten geteilt werden, scheint auch 2019 noch aktuell. Es ist zu hoffen, dass sich durch die Open Data-Policy des FWF, durch die derzeit im Aufbau befindliche Forschungsdatenmanagement-Policy an den österreichischen Universitäten sowie durch die Tatsache, dass mit der Errichtung von AUSSDA nunmehr auch in Österreich ein professionell arbeitendes sozialwissenschaftliches Datenarchiv zur Verfügung steht, das Bewusstsein für die Sinnhaftigkeit und die Bereitschaft zu einer adäquaten Datenarchivierung in den kommenden Jahren verbessert.

In Hinblick auf die Zahl der potenziellen NutzerInnnen der bei AUSSDA archivierten Daten ergibt unsere Erhebung folgendes Bild: An den untersuchten Universitäten arbeiten derzeit etwa 800 bis 1000 beschäftigte SozialwissenschaftlerInnen, die selbst empirische Forschungsprojekte durchführen und/oder Lehrveranstaltungen in empirischer Sozialforschung abhalten. Beide Gruppen, sowohl Forschende als auch Lehrende, benötigen für ihre Arbeit immer wieder empirische Daten mit Österreichbezug und werden daher gerne auf entsprechende Daten zurückgreifen, wenn diese im Archiv vorhanden sind (bisher griffen viele BerufskollegInnen zu diesem Zweck meist auf die Angebote der deutschen GESIS zurück). Neben dieser primären Nutzergruppe könnte auch ein Teil der ca. 2000 universitären SozialwissenschaftlerInnen, die selbst keine empirischen Forschungen durchführen und auch nicht in diesem Bereich lehren, in bestimmten Fällen Interesse an archivierten Forschungsdaten haben. Ein weiteres Nutzerpotenzial an den Universitäten ist die Gruppe der Studierenden, die im Rahmen von Masterarbeiten und Dissertationen empirische Daten mit Österreichbezug benötigen.

Die Archivierung sozialwissenschaftlicher Forschungsdaten in einem österreichischen Datenarchiv erscheint somit aus mehreren Perspektiven als sinnvoll: WissenschaftlerInnen können ihre Sichtbarkeit und ihren Impact in der österreichischen Forscher-Community erhöhen, neue Kooperationsmöglichkeiten finden und weitere, über Fachgrenzen hinausgehende Kanäle für ihren wissenschaftlichen Output erschließen (Bauer et al. 2015b, S. 571). Studierende könnten von den Lehren- 
den besser und gezielter an die in Österreich durchgeführte Forschung herangeführt werden und im Studium oder bei Qualifizierungsarbeiten auf hochqualitative Daten zurückgreifen.

Funding Open access funding provided by Johannes Kepler University Linz.

Open Access Dieser Artikel wird unter der Creative Commons Namensnennung 4.0 International Lizenz (http://creativecommons.org/licenses/by/4.0/deed.de) veröffentlicht, welche die Nutzung, Vervielfältigung, Bearbeitung, Verbreitung und Wiedergabe in jeglichem Medium und Format erlaubt, sofern Sie den/die ursprünglichen Autor(en) und die Quelle ordnungsgemäß nennen, einen Link zur Creative Commons Lizenz beifügen und angeben, ob Änderungen vorgenommen wurden.

\section{Literatur}

Baack, Stefan. 2015. Datafication and empowerment: How the open data movement re-articulates notions of democracy, participation, and journalism. Big Data \& Society 2:1-11. https://doi.org/10.1177/ 2053951715594634.

Bauer, Bruno, Andreas Ferus, Juan Gorraiz, Veronika Gründhammer, Christian Gumpenberger, Nikolaus Maly, Johannes Michael Mühlegger, José Luis Preza, Barbara Sanchéz Solís, Nora Schmidt, und Christian Steineder. 2015a. Forschende und ihre Daten. Ergebnisse einer österreichweiten Befragung - Report 2015. Version 1.2. Wien: Eigenverlag.

Bauer, Bruno, Andreas Ferus, Juan Gorraiz, Veronika Gründhammer, Christian Gumpenberger, Nikolaus Maly, Johannes Michael Mühlegger, José Luis Preza, Barbara Sanchéz Solís, Nora Schmidt, und Christian Steineder. 2015b. Forschende und ihre Daten: Ergebnisse einer österreichweiten Befragung. Report 2015 - Executive Summary und Empfehlungen. Mitteilungen der VÖB 68(3-4):566-579.

BMFWF. 2017. Geisteswissenschaften Sozialwissenschaften Kulturwissenschaften. Beitrag zur strategischen Weiterentwicklung der Rahmenbedingungen. Wien: Bundesministerium für Wissenschaft, Forschung und Wirtschaft.

Borgman, Christine. 2012. The conundrum of sharing research data. Journal of the Association for Information Science and Technology 63(6):1059-1078. https://doi.org/10.1002/asi.22634.

Couldry, Nick. 2014. Inaugural: a necessary disenchantment: myth, agency and injustice in a digital world. The Sociological Review 4:880-897.

Fecher, Benedikt, Sascha Friesike, und Marcel Hebing. 2015. What drives academic data sharing? PLoS ONE 10(2):e118053. https://doi.org/10.1371/journal.pone.0118053.

FWF. 2018. Open access policy. https://www.fwf.ac.at/de/forschungsfoerderung/open-access-policy/. Zugegriffen: 20. Dez. 2018.

Heidorn, P. Bryan. 2008. Shedding light on the dark data in the long tail of science. Library Trends 57(2):280-299.

Lycett, Mark. 2013. 'Datafication': making sense of (big) data in a complex world. European Journal for Information Systems 4:381-386. https://doi.org/10.1057/ejis.2013.10.

Prandner, Dimitri, und Lena Raffetseder. 2018. Lücken schließen und Erkenntnisse sichern - AUSSDA als Infrastruktureinrichtung für die österreichischen Sozialwissenschaften. SWS Sozialwissenschaftliche Runderschau 58(2):237-243.

Dimitri Prandner ist wissenschaftlicher Mitarbeiter von AUSSDA - The Austrian Social Science Data Archive am Institut für Soziologie an der Johannes-Kepler-Universität Linz und lehrt als Senior Lecturer Methoden der empirischen Sozialforschung an der Paris-Lodron-Universität Salzburg. Seine Forschungsschwerpunkte liegen in den Bereichen der Nachrichtensoziologie, politischen Soziologie und quantitativen Umfrageforschung.

Otto Bodi-Fernandez wissenschaftlicher Mitarbeiter von AUSSDA - The Austrian Social Science Data Archive am Center for Social Research der Universität Graz. Aufgrund seiner Spezialisierung auf empirische Methoden und Datenanalyse war er in verschiedenen Forschungsprojekten unterschiedlicher Disziplinen tätig. Seine Forschungsinteressen liegen in den Bereichen soziale Ungleichheit, Bildung und Kindheitsforschung. 
Franz Höllinger ist a.o. Universitätsprofessor am Institut für Soziologie der Universität Graz. Er arbeitet seit 1986 am Sozialen Survey Österreich und am International Social Survey Programme mit. Seine Arbeitsschwerpunkte liegen in den Bereichen Religionssoziologie, Familiensoziologie und international vergleichende Sozialforschung.

Ahmed Tabakovic BA, ist studentischer Mitarbeiter am Fachbereich Kommunikationswissenschaft der Universität Salzburg und war als Projektmitarbeiter bei AUSSDA - The Austrian Social Science Data Archive tätig. Sein aktuelles Forschungsinteresse liegt im Bereich der Reputationsforschung. Im Rahmen seiner Masterarbeit analysiert er die Reputation von religiösen Institutionen. 